\title{
Genomic diversity of the human intestinal parasite Entamoeba histolytica
}

\author{
Gareth D Weedall', C Graham Clark², Pia Koldkjaer', Suzanne Kay ${ }^{1}$, Iris Bruchhaus³, Egbert Tannich³, \\ Steve Paterson ${ }^{1}$ and Neil Hall ${ }^{1 *}$
}

\begin{abstract}
Background: Entamoeba histolytica is a significant cause of disease worldwide. However, little is known about the genetic diversity of the parasite. We re-sequenced the genomes of ten laboratory cultured lines of the eukaryotic pathogen Entamoeba histolytica in order to develop a picture of genetic diversity across the genome.

Results: The extreme nucleotide composition bias and repetitiveness of the E. histolytica genome provide a challenge for short-read mapping, yet we were able to define putative single nucleotide polymorphisms in a large portion of the genome. The results suggest a rather low level of single nucleotide diversity, although genes and gene families with putative roles in virulence are among the more polymorphic genes. We did observe large differences in coverage depth among genes, indicating differences in gene copy number between genomes. We found evidence indicating that recombination has occurred in the history of the sequenced genomes, suggesting that E. histolytica may reproduce sexually.
\end{abstract}

Conclusions: E. histolytica displays a relatively low level of nucleotide diversity across its genome. However, large differences in gene family content and gene copy number are seen among the sequenced genomes. The pattern of polymorphism indicates that E. histolytica reproduces sexually, or has done so in the past, which has previously been suggested but not proven.

\section{Background}

Entamoeba histolytica is a parasite of the human large intestine, commonly contracted by ingesting contaminated water or food. The parasite has a two stage lifecycle consisting of a cyst, the infective stage outside of the host, and a trophozoite, the reproductive stage within the host. Invasive amoebiasis results when trophozoites attack the gut wall, leading to diarrhoea, dysentery and in some cases dissemination to organs (commonly the liver) where abscesses result [1]. If untreated, amoebic liver abscess can be fatal.

E. histolytica infection is endemic in many parts of the world where sanitation infrastructure is poor; elsewhere infection tends to be restricted to certain at-risk groups such as residents in institutions for the mentally handicapped and men who have sex with men [2]. In endemic areas, exposure can be extremely high: an annual

\footnotetext{
* Correspondence: neil.hall@liv.ac.uk

'Institute of Integrative Biology, University of Liverpool, Biosciences building, Crown Street, Liverpool L69 7AH, UK

Full list of author information is available at the end of the article
}

incidence of $40 \%$ was estimated among children in an urban slum in Bangladesh [3]. The global prevalence of infection was estimated to be approximately $10 \%$ of the world's population in the 1980s [4]. Of these, approximately $90 \%$ were estimated to be asymptomatic carriers while $10 \%$ developed invasive amoebiasis, leading to 100,000 deaths per year [4]. This estimate was made prior to the redescription in 1993 of E. histolytica as two separate species, E. histolytica and E. dispar [5]. E. histolytica causes invasive amoebiasis while $E$. dispar is avirulent, and it was thought that $E$. dispar infection might account for the predominance of asymptomatic carriers. However, even considering only E. histolytica infections, invasive amoebiasis appears still to be a relatively rare outcome of infection.

Understanding the nature of amoebic virulence motivates a substantial body of research. Several lines of evidence suggest that genetic factors of the parasite affect its virulence: the morphologically identical but genetically distinct $E$. dispar appears to be avirulent; distinct E. histolytica genotypes have been detected in liver 
abscess and feces from the same infected person [6]; different endemic populations show different rates of liver abscess [7]; a short-term transmission chain of virulent parasites has been reported [8].

The E. histolytica genome has been sequenced to a draft level with 12.5-fold coverage genome assembly consisting of approximately 24 megabases in 888 scaffolds [9]. Subsequent reassembly with additional data produced a genome assembly of approximately 21 megabases in 1,496 scaffolds [10]. The ploidy of E. histolytica is uncertain though some studies suggest that it may be tetraploid [11]. As part of the effort to identify genetic determinants of virulence and to gain an understanding of the diversity of the parasite, we re-sequenced the complete genomes of a number of $E$. histolytica strains. We show that single nucleotide diversity is relatively low among these parasites, but that gene copy number variation may be a significant contributor to genome diversity. We also provide evidence that $E$. histolytica may reproduce sexually and define a set of high quality SNPs that could be used as markers for genotyping $E$. histolytica isolates.

\section{Results and discussion}

\section{Sequencing and mapping of Entamoeba strains to the HM-1:IMSS reference genome}

Eight strains of E. histolytica derived from infected humans and adapted to axenic laboratory culture, as well as two clones of different virulence phenotypes derived from the axenic strain HM-1:IMSS, were sequenced and mapped to the published genome sequence of the HM-1:IMSS strain. Details of the origins of each strain are shown in Table 1. All sequence data were submitted to the European Nucleotide Archive under the following accessions: ERS132616 (HM-1A); ERS132617 (HM-1B); ERS132618 (Rahman); ERS132619 (2592100); ERS132620 (PVBM08B); ERS132621 (PVBM08F); ERS132622 (IULA:1092:1); ERS132623

\begin{tabular}{llll}
$\begin{array}{l}\text { Table 1 Entamoeba histolytica } \\
\text { study }\end{array}$ \\
\hline Strain & Origin & Date & Symptoms \\
\hline HM-1A & Mexico & 1967 & Intestinal amoebiasis \\
HM-1B & Mexico & 1967 & Intestinal amoebiasis \\
Rahman & UK & 1964 & Asymptomatic \\
2592100 & Bangladesh & 2005 & Intestinal amoebiasis \\
PVBM08B & Italy & 2007 & Intestinal amoebiasis \\
PVBM08F & Italy & 2007 & Intestinal amoebiasis \\
IULA:1092:1 & Venezuela & 1992 & Intestinal amoebiasis \\
HK-9 & Korea & 1951 & Intestinal amoebiasis \\
MS84-1373 & Bangladesh & 2006 & Asymptomatic \\
MS27-5030 & Bangladesh & 2006 & Asymptomatic \\
\hline
\end{tabular}

asolated from a sailor, ultimate origin unknown.
(HK-9); ERS132624 (MS84-1373); ERS132625 (MS275030).

The E. histolytica genome is known to be very $\mathrm{A}+\mathrm{T}$ rich (approximately $75 \% \mathrm{~A}+\mathrm{T}$ ) and repetitive, which both make sequence alignment difficult. In order to assess this repetitiveness and estimate how much unique mapping we might expect from short sequencing reads, we made a set of $50 \mathrm{bp}$ reads from the reference genome and mapped them to the reference: $72.36 \%$ aligned to only one location, $12.46 \%$ aligned to two different locations, $10.83 \%$ aligned to between 3 and 10 locations and $4.35 \%$ aligned to more than 10 locations. As reads were derived from the reference genome, these proportions relate directly to proportions of the genome, so we expected up to 14 megabases of sequence to be unique enough for unique alignment to 50 bp reads. This shows that the majority of the reference genome is unique enough for accurate alignment of $50 \mathrm{bp}$ reads. When we aligned the read libraries, we saw that approximately $75 \%$ of reads that aligned uniquely and approximately 13 megabases of the reference genome were covered by uniquely aligned reads, broadly consistent with our expectations.

Table 2 shows statistics relating to read alignment and SNP calling in the strains. Coverage of the genome was generally much better across coding sequences than across intergenic regions and the proportion of each gene covered was generally high (Additional file 1). The poorer coverage of intergenic regions could be due to their repetitiveness and their more extreme nucleotide composition bias towards $\mathrm{A}$ and $\mathrm{T}$, as sequencing libraries can be biased toward more balanced nucleotide compositions.

SNP calling was carried out, using thresholds for minimum and maximum coverage and minimum mapping quality. The number of sites passing these thresholds ranged from approximately 9 to 14 megabases. At these positions, bases were predicted and SNPs called (Additional file 2). SNP calls for heterozygotes will be affected by the unknown ploidy (a ploidy of 4 was assumed based on the estimate of Willhoeft and Tannich [11]). At least $89.55 \%$ of the homozygous SNPs in the Rahman strain were also called as variants when sequenced independently using different sequencing technology (454 sequencing; Additional file 3). However, as few as $43.02 \%$ of the heterozygous SNPs were similarly validated. It is difficult to ascertain whether the unvalidated SNPs are false positives or are due to the lower coverage of the 454 sequencing. Due to the greater uncertainty associated with heterozygous SNP calls, homozygous SNP calls showing inter-strain variation and a base call in every strain were tabulated as a set of 'high quality candidate markers' (Additional file 4). These SNPs were used to infer a phylogeny of sequenced strains and to test for evidence of meiotic recombination. 
Table 2 Alignment and SNP-calling statistics

\begin{tabular}{lccccccc}
\hline Strain & Reads $^{\mathbf{a}}$ & c50, $^{\text {c95 } \text { cov }^{\mathbf{b}}}$ & SNP-able sites & SNP & Hom $^{\mathbf{d}}$ & Het $^{\mathbf{e}}$ & Divergence (hom only) $^{\mathbf{f}}$ \\
\hline HM-1A & $13,743,406$ & 35,141 & $10,012,951$ & 2,217 & 229 & 1,988 & $0.22(0.02)$ \\
HM-1B & $9,586,924$ & 26,95 & $9,819,882$ & 1,995 & 220 & 1,775 & $0.20(0.02)$ \\
Rahman & $19,498,380$ & 32,198 & $9,817,503$ & 6,889 & 3,767 & 3,122 & $0.70(0.38)$ \\
2592100 & $13,560,609$ & 26,127 & $10,025,805$ & 6,788 & 3,128 & 3,660 & $0.68(0.31)$ \\
PVBM08B & $17,627,870$ & 36,172 & $10,335,217$ & 7,999 & 4,225 & 3,774 & $0.77(0.41)$ \\
PVBM08F & $8,436,907$ & 19,65 & $10,253,328$ & 6,602 & 3,613 & 2,989 & $0.64(0.35)$ \\
IULA:1092:1 & $19,041,335$ & 48,155 & $11,934,434$ & 10,014 & 4,897 & 5,117 & $0.84(0.41)$ \\
HK-9 & $21,193,087$ & 41,202 & $10,678,584$ & 9,155 & 4,428 & 4,727 & $0.86(0.41)$ \\
MS84-1373 & $21,479,273$ & 51,209 & $10,308,534$ & 8,373 & 4,027 & 4,346 & $0.81(0.39)$ \\
MS27-5030 & $20,403,218$ & 47,225 & $8,731,329$ & 7,001 & 3,302 & 3,699 & $0.80(0.38)$ \\
\hline
\end{tabular}

${ }^{a}{ }^{\prime}$ Reads' $=$ the number of uniquely aligned reads. ${ }^{b}{ }^{\prime} \mathrm{c} 50, \mathrm{c95} \mathrm{cov}^{\prime}=$ the 50th and 95th centile of coverage depth. ${ }^{\mathrm{C}}$ SNP-able sites' $=$ positions passing criteria for allowing a SNP to be called (see Materials and methods). ${ }^{d \prime} \mathrm{Hom}^{\prime}=$ homozygous SNP. ${ }^{\mathrm{e}} \mathrm{Het}^{\prime}=$ heterozygous SNP. ${ }^{\mathrm{f}}$ Divergence (hom only) ${ }^{\prime}=$ divergence from reference genome (divergence calculated using only homozygous SNPs).

\section{Genealogy of $E$. histolytica strains}

Figure 1 shows the relationship among the sequenced strains. In this phylogeny the three strains derived from Bangladesh cluster together. The HM-1:IMSS-derived clones HM-1A and HM-1B cluster very closely with HM-1:IMSS. The pair of strains isolated from feces (PVBM08F) and from colonic biopsy (PVBM08B) from the same patient also cluster very closely together. The geographical origin of this pair of isolates is not known, but the patient from whom they were isolated had visited both Colombia and Liberia prior to the strains being isolated. These strains form an outgroup to the other strains when the phylogeny is rooted relative to $E$. dispar. HK-9 (isolated in Korea) appears to be rather different to the other strains. Rahman and IULA:1092:1 cluster very closely together. Rahman was originally isolated from a sailor in the UK, so its ultimate geographical origin is unknown. IULA:1092:1 was isolated in Venezuela.

\section{Evidence of meiotic recombination among E. histolytica strains}

Under the infinite sites model of evolution, nucleotide sites mutate only once in the evolutionary history of a sample of related sequences. Given this, the occurrence of four haplotypes due to recombination should be

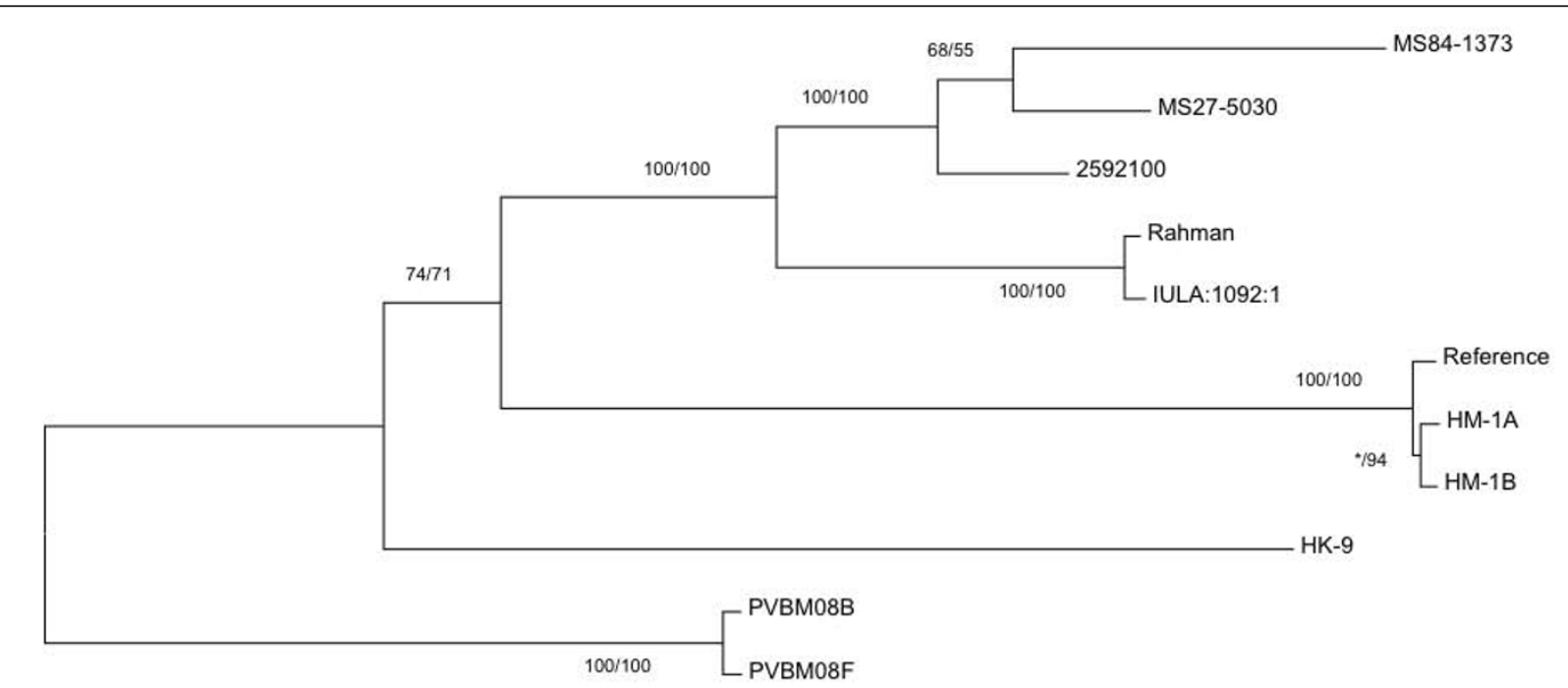

Figure 1 Relationships among sequenced E. histolytica strains. Relationships were estimated using 3,696 polymorphic sites and two phylogenetic approaches, distance-based analysis (neighbor-joining) and maximum likelihood (ML), both as implemented in MEGA 5 [52]. ML used the General Time Reversible model of evolution, selected as best using the model testing function in MEGA 5, while neighbor-joining used the maximum composite likelihood model. Statistical support for distance and ML trees was evaluated using bootstrapping (1,000 replicates). The ML tree is shown. The tree is rooted by comparison with orthologous positions in E. dispar (data not shown) along the branch to the PVBM08B and PVBM08F strains. Bootstrap values from both types of phylogenetic analysis are shown in the following order: Distance/ML. An asterisk indicates where the bootstrap value is under $50 \%$. 
more common for distant pairs of polymorphic positions as the probability of a recombination event increases with distance. Random sampling of pairs of polymorphic sites on the same reference genome scaffold showed that the proportion showing four haplotypes was significantly positively correlated with distance (Figure 2; Spearman's rho $=0.83, P<2.2 \times 10^{-16}$ ). The average distance between sites forming two haplotypes (indicating linkage disequilibrium among sites) was $18,485 \mathrm{bp}$, between sites forming three haplotypes was 46,644 bp and between sites forming four haplotypes (indicating a recombination event) was $60,176 \mathrm{bp}$.
Among polymorphic sites in the same genomic scaffold (that is, physically linked in the genome), the proportion of two-haplotype pairs was $23.2 \%$, three-haplotype pairs $68.3 \%$ and four-haplotype pairs $8.5 \%$. In contrast, among polymorphic sites in different genomic scaffolds, the proportion of two-haplotype pairs was markedly lower $(7.2 \%)$, while the proportions of three- and four-haplotype pairs were higher ( $81.1 \%$ and $11.7 \%$, respectively), reflecting a reduction in linkage disequilibrium among sites on different chromosomes. Physically unlinked sites would be expected to remain in linkage disequilibrium in strictly asexual lineages. In order to support our

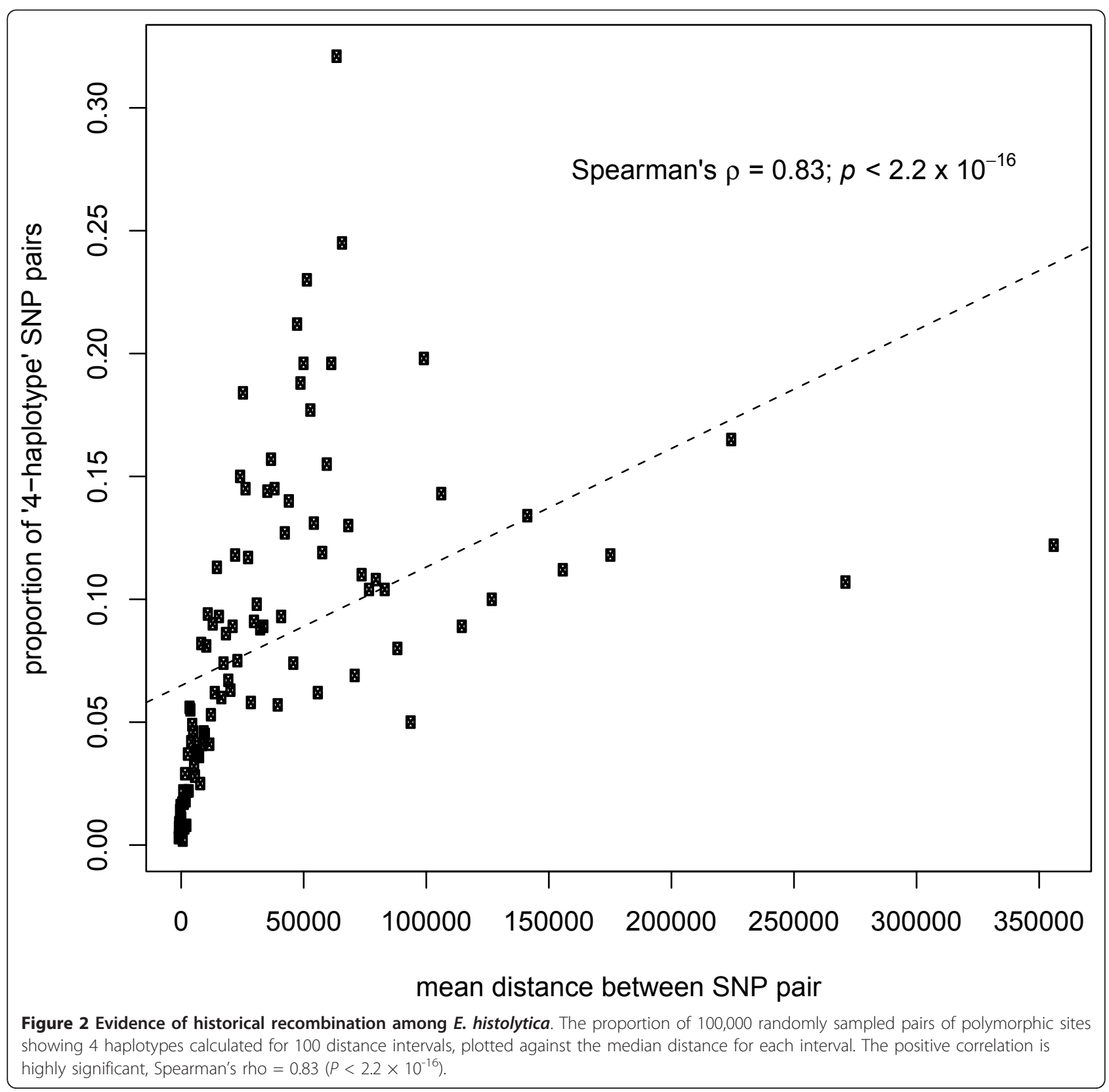


inference of recombination in the history of the sequenced strains, we simulated the expected effects of recombination and of the analogous process of gene conversion upon the test. The results showed that, as the rate of recombination increases, the proportion of pairs of SNP showing all four possible haplotypes increases with distance (with a significant positive correlation). However, when gene conversion is modeled, the proportion of 'four-haplotype pairs' is not significantly positively correlated with distance (Additional file 3). Taken together, these results strongly suggest that sexual reassortment of chromosomes and meiotic recombination have occurred in the history of these genomes. Genetic data from a single, putatively panmictic, population will be required to confirm this result and to estimate the frequency of recombination events.

\section{SNPs among E. histolytica strains}

Overall genetic diversity appears to be rather low among these genomes. For these data it is difficult to make an estimate of diversity such as the average pairwise divergence because both the ploidy and the phase of variant bases are unknown. Also, all data are mapped to a single reference genome, so comparisons to each other may not account for differences in genome coverage. Therefore, we estimated the divergence of each genome from the HM-1:IMSS reference genome as the proportion of sites that could be called as SNPs that were called as SNPs. We used both the number of putative homozygous SNPs and the total number of both homozygous and heterozygous SNPs to estimate a lower and an upper boundary for divergence. The estimates are shown in Table 2. Divergence estimates counting only putative homozygous positions were between 0.3120 and 0.4147 SNPs per kilobase, while estimates counting both putative homozygous and heterozygous positions were between 0.6439 and 0.8573 SNPs per kilobase.

All SNP calls estimated for each strain were tabulated and can be viewed in Additional file 2. In order to identify polymorphic genes that may be under diversifying selection, the numbers of nonsynonymous and synonymous homozygous SNPs per gene (compared to the HM-1:IMSS reference genome) were counted and tabulated in Additional file 5. Putative nonsense mutations in genes are tabulated in Additional file 6. In these Additional files, only homozygous variants were counted in order to reduce the chance of counting false heterozygous SNPs caused by alignment of paralogous sequence. Genes with five or more nonsynonymous homozygous SNPs in one or more strain are tabulated in Additional file 7. This rather crude method was used instead of estimating $\mathrm{dN}$ (nonsynonymous divergence per nonsynonymous site) and dS (synonymous divergence per synonymous site), despite its bias towards longer genes and genes with no closely related paralogues (that is, genes with a greater proportion of unique coverage), because the small number of variant positions per gene makes $d N$ and $d S$ estimates very sensitive to small stochastic differences in the number of variants and not robust. Fifty-three genes contained five or more homozygous nonsynonymous SNPs relative to HM-1: IMSS. Among these genes are several encoding proteins involved in host-parasite interactions, including the intermediate chains of the galactose/N-acetyl-D-galactosamine-inhibitable lectin (Gal/GalNAc lectin) igl1 (EHI_006980) and igl2 (EHI_065330), which were divergent in the HK-9 strain, the light chain Gal/GalNAc lectin gene $l g l$ (EHI_035690), which was divergent in the Rahman and IULA:1092:1 strains, and three serinethreonine-isoleucine-rich protein (STIRP) genes (EHI_073630, EHI_004340 and EHI_012330). The Gal/ GalNAc lectin complex is a major virulence factor expressed on the surface of the trophozoite that has multiple functions, including binding to the mucus layer and to epithelial cells and involvement in host cell killing [12]. STIRPs are putative trophozoite surfaceexpressed proteins, some or all of which bind to host epithelial cells and cause cell death [13].

The gene that displayed most polymorphism, the STIRP gene EHI_073630, lay on the HM-1:IMSS scaffold DS571171. The gene is annotated as a STIRP, with predicted amino-terminal signal peptide and a carboxy-terminal transmembrane domain, but is sufficiently distantly related to other described STIRPs [13] that reads aligned uniquely across its entire length, unlike in other STIRP genes (data not shown). Across scaffold DS571147, the majority of polymorphic sites were in this gene and were homozygous in each strain. In addition, HM-1:IMSS and its derivatives HM-1A and HM-1B displayed a haplotype that differed greatly from the other strains, which were generally similar to each other. This pattern of polymorphism appears to break down outside of the gene (Figure 3). The HM-1-like allele differs from the others by 27 to 34 homozygous synonymous SNPs and 48 to 60 homozygous nonsynonymous SNPs across its $15,210 \mathrm{bp}$. Given an estimated 1,896 theoretically synonymous and 13,311 theoretically nonsynonymous sites, the estimated divergence values are $\mathrm{dS}=0.014$ to 0.017 and $\mathrm{dN}=0.0036$ to 0.0045 $(\mathrm{dN} / \mathrm{dS}=0.20$ to 0.32$)$. The relatively high value of $\mathrm{dS}(14$ to 17 variants per kilobase) suggests that the alleles my have diverged a long time ago, consistent with a pattern of long-term allelic dimorphism as seen in some Plasmodium genes encoding surface-expressed proteins that interact with host cells [14,15].

\section{Gene copy number variation among E. histolytica strains}

Another source of genomic diversity in addition to SNPs is variation in the number of paralogous copies of genes 


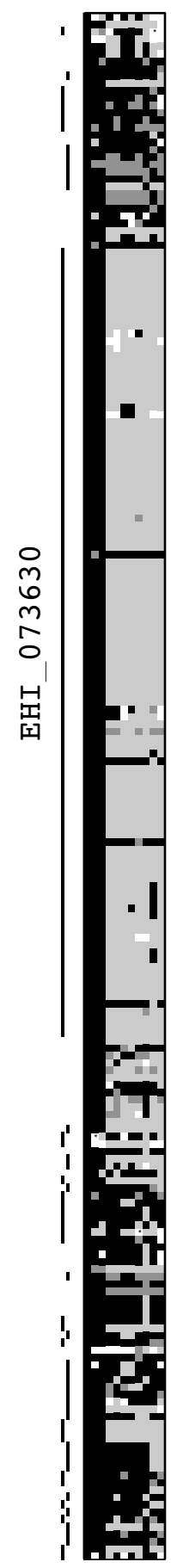

Figure 3 The pattern of polymorphism across scaffold DS571171. Polymorphic sites on scaffold DS571171 are represented by shaded squares: black for bases that match the HM-1:IMSS reference; light grey for bases that differ from the reference; dark grey for heterozygous positions; white for positions where no base was assigned. Three positions (marked with asterisks) had an additional variant base in one strain. Each row represents a strain in the following order from top to bottom: reference sequence (HM-1: IMSS), HM-1A, HM-1B, Rahman, 2592100, PVBM08B, PVBM08F,

IULA:1092:1, HK-9, MS84-1373, MS27-5030. Sites in coding sequences are marked with black lines above the figure. and the content of gene families. To analyze this, depth of coverage of genes was compared between strains. While each strain showed a proportion of genes with high coverage, these were not the same genes in all strains. Figure 4 shows that strains varied considerably in the copy number of different genes, even putative close relatives such as PVBMOB and PVBMOF. This pattern remained generally consistent when all uniquely mapped reads were considered or only uniquely mapped reads with unique start positions were considered, although the signal was reduced overall and for some genes was lost.

Genes with coverage depth more than twofold the average of $\mathrm{HM}-1 \mathrm{~A}$ and $\mathrm{HM}-1 \mathrm{~B}$ in one or more strain were designated putative high copy number genes (Additional file 8). Putative missing genes (reads per kilobase per million mapped reads (RPKM) $<1$ and $<$ $50 \%$ of the average of HM-1A and HM-1B) were also tabulated (Additional file 9). Among the putative high copy number genes in one or more strain were genes encoding ribosomal proteins (EHI_081320, EHI_104660, EHI_108500, EHI_124300, EHI_124330, EHI_137870, EHI_152080, EHI_152570, EHI_152610, EHI_153070, EHI_179980, EHI_181560, EHI_182920, EHI_193330, EHI_199970). Also represented were members of large gene families encoding AIG1 (avirulence induced gene 1)-like proteins (EHI_022500, EHI_079610, EHI_115170, EHI_126560, EHI_144270, EHI_157260, EHI_176590, EHI_195250, EHI_195260), BspA (Bacteroides forsythus surface protein A)-like leucine rich repeat-containing proteins (EHI_002120, EHI_005660, EHI_008340, EHI_049160, EHI_094080, EHI_102380, EHI_113190, EHI_123820, EHI_137910, EHI_147680, EHI_163960, EHI_189090, EHI_191510, EHI_192600) and ariel family proteins (EHI_028430, EHI_057430, EHI_080200). Among the genes absent from different strains were genes on a duplicated genome region previously described in HM-1:IMSS [10], indicating variation in the copy number of these duplications among strains. Many putative missing genes were unannotated, and a proportion of them may be incorrect gene models. The results suggest that strains vary in their gene family content and that duplication and loss of members of these large gene families is a dynamic process.

A region of high coverage occurring only in the Rahman strain and spanning several genes, encoding $60 \mathrm{~S}$ ribosomal protein L38 (EHI_023840), a hypothetical protein (EHI_023850), protein kinase domain containing protein (EHI_023860), WD domain containing protein (EHI_023870), ubiquitin-conjugating enzyme family protein (EHI_023880), nuclear movement protein (EHI_023890) and hypothetical protein (EHI_023900), is likely to represent a segmental duplication similar to those described in HM-1:IMSS [10]. Data from an 

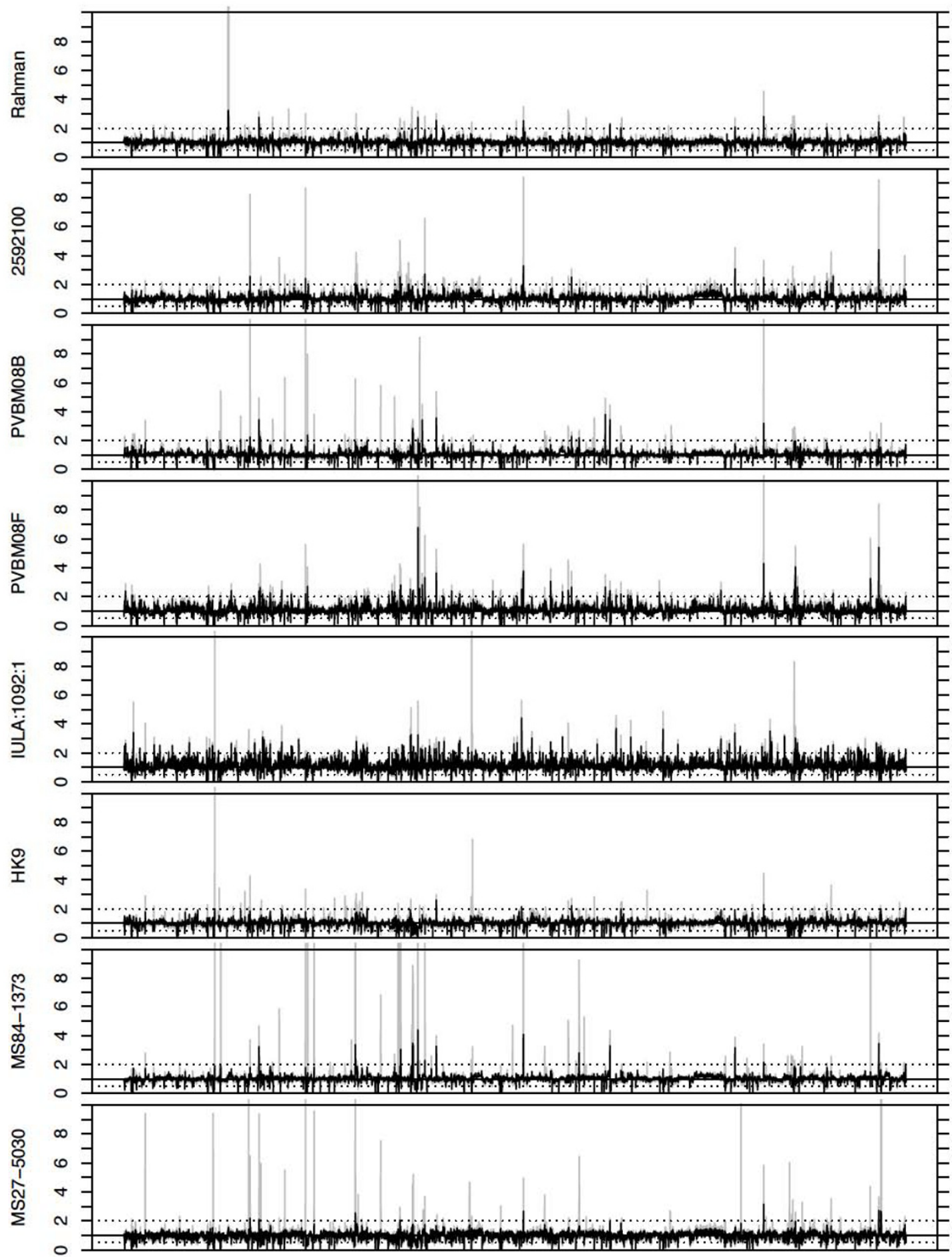

Figure 4 Copy number variation among E. histolytica genes. Coverage depth of a set of 6,228 E. histolytica genes relative to HM-1:IMSS in eight sequenced strains. The $y$-axis displays the ratio of a strain's RPKM to the average RPKM of HM-1A and HM-1B. The $x$-axis represents 6,228 annotated E. histolytica genes ordered by their amoebaDB gene ID. Ratios greater than 10 were truncated for display purposes. Grey bars represent results for all uniquely aligned reads, black bars for uniquely aligned reads with unique start positions on the reference genome (see Materials and methods). 
independently generated sequencing library of the Rahman strain sequenced by 454 sequencing (manuscript in preparation) confirms this putative segmental duplication (Figure 5). The duplicated genome region occurs adjacent to a repetitive element (Entamoeba repeat element 2, ERE2) and long interspersed nuclear elements (LINE1 and LINE2), which also mark a break in synteny between $E$. histolytica and $E$. dispar (data not shown). An approximate estimate of the number of copies of the duplicated region was made by comparing coverage depth in Rahman to that in HM-1B at sites in the unduplicated region (positions 1 to 21,000) and the duplicated region (positions 21,000 to 33,380 ). In the unduplicated region, the median ratio of coverage in Rahman to HM-1B was 1.1 and the 25th and 75th centiles were 0.7 and 1.6. In the duplicated region the median was 25.0 and the 25 th and 75 th centiles were 12.8 and 34.8. A similar putative segmental duplication in MS84-1373 spans a number of genes encoding unknown proteins (EHI_072460, EHI_072470, EHI_072480, EHI_072490, EHI_072500, EHI_072510, EHI_072520, EHI_072530).

\section{Conclusions}

We report the genome-wide diversity of the enteric parasite E. histolytica. Overall, diversity among the sequenced genomes was rather low: divergence from the reference genome was between 0.312 and 0.857 SNPs per kilobase, depending on whether heterozygous SNPs were counted or not. An analogous measure of genomic diversity in Plasmodium falciparum estimated divergence from the reference strain 3D7 of a clinical isolate from Ghana (divergence $=1.31$ SNPs per kilobase); of a strain (IT) derived from central America (divergence = 1.10 SNPs per kilobase); and divergence between P. falciparum 3D7 and the related species Plasmodium reichenowi of 20.30 SNPs per kilobase [16]. Previous studies of genetic diversity among $E$. histolytica isolates have produced conflicting results. In general, studies using repetitive DNA as markers of diversity, such as the serine-rich E. histolytica protein (SREHP) gene and the short tandem repeats separating tRNA genes, have reported high diversity among genomes [17-21]. In contrast, studies of SNPs, though few in number, have reported minimal diversity [22-24]. Our results agree with the latter studies, suggesting that SNP is low in $E$. histolytica. However, our results also suggest that recombination and reassortment of alleles may have shuffled these alleles to create new haplotypes. Therefore, recombination and reassortment may be relatively more important than point mutation in generating diversity among parasite lineages.

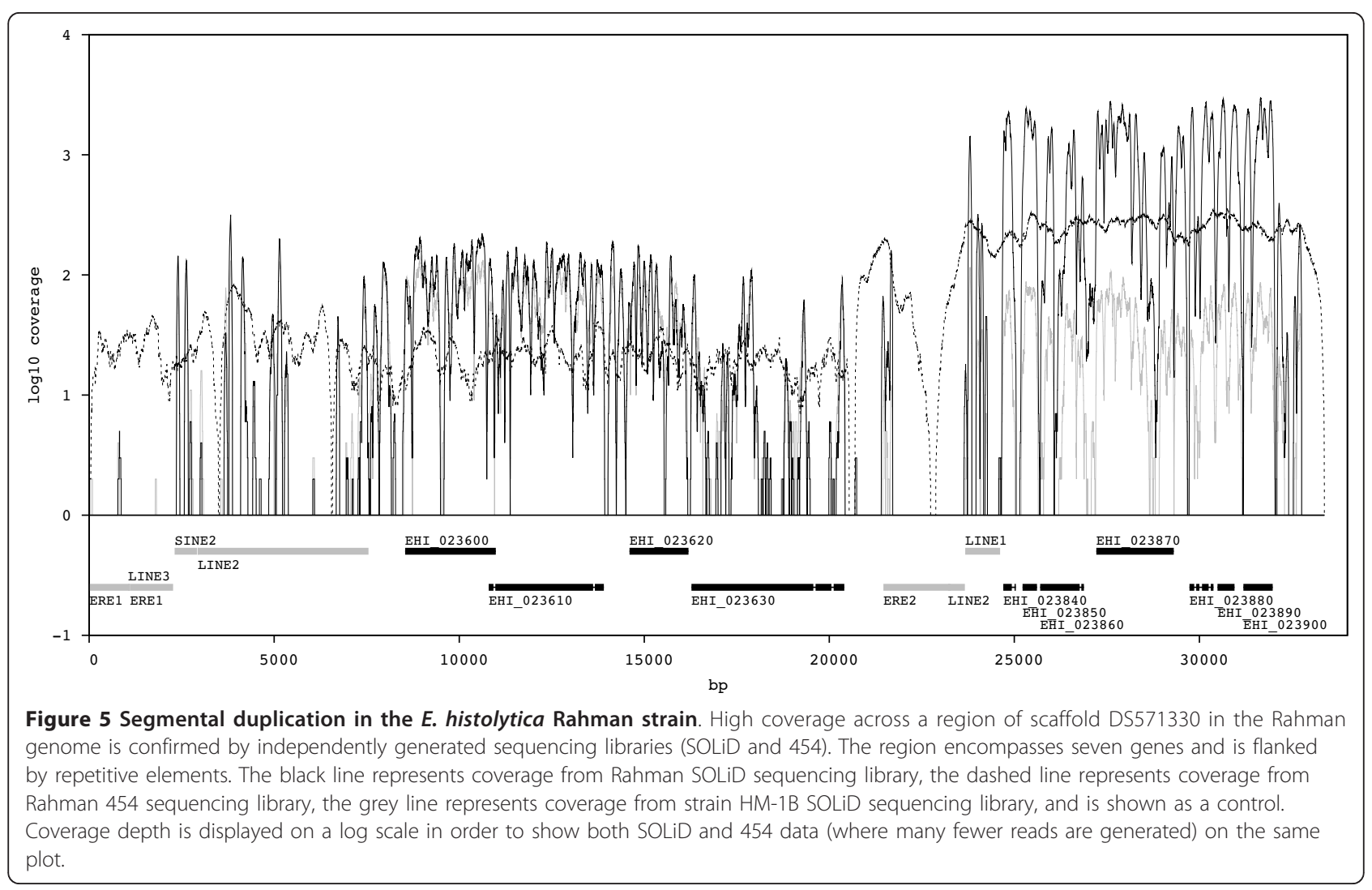


The highly polymorphic STIRP gene EHI_073630 showed a pattern of polymorphism consistent with allelic dimorphism, as described in P. falciparum. It is difficult to say with certainty that the gene is truly dimorphic since it is a member of a gene family so it is possible that the aligned sequences are paralogous rather than allelic. However, the alignment clearly shows that the HM-1-like form of the gene is present in HM$1 \mathrm{~A}$ and $\mathrm{HM}-1 \mathrm{~B}$ and not present in the other strains. The causes and significance of allelic dimorphism in $P$. falciparum are not fully understood, but several genes that show this pattern of polymorphism (merozoite surface proteins MSP-1 [14], MSP-2 [25,26], MSP-3 [27], and MSP-6 [28], and erythrocyte binding antigen EBA175 [29]) are involved in host cell invasion. Immune recognition of some of these proteins has been associated with protection [30-33], and a proportion of antibodies may recognize allele-specific epitopes [31,33,34]. Whether any of this applies to the E. histolytica gene EHI_073630 remains to be seen. More extensive sampling of this region of the genome from more parasite isolates might indicate whether or not EHI_073630 is truly dimorphic and if the HM-1-like form of the gene is geographically restricted to Mexico, or segregates in other populations.

Sexual reproduction has not been observed in Entamoeba. However, since the publication of the genome of the E. histolytica HM-1:IMSS strain showed that it possesses the majority of a set of genes involved in meiosis, it has been suggested that E. histolytica may reproduce sexually $[9,35,36]$. Gene conversion, a process involving homologous recombination, appears to have occurred among paralogous genes in the E. histolytica and E. dispar lineages [37]. The evidence from patterns of polymorphism presented here suggests that historical recombination events have broken up linkage between polymorphic sites and linkage has declined with increasing distance between sites on the same chromosome, consistent with the occurrence of meiotic recombination. Taken together, these lines of evidence strongly suggest that sexual reproduction has occurred in E. histolytica and adds it to the list of sexual protozoal parasites previously believed to be clonal, such as species of Leishmania [38] and Giardia [39]. Whether sexual reproduction is common in parasite populations could be tested by genotyping large numbers of individuals from endemic populations and measuring linkage disequilibrium.

Large differences in the depth of coverage among genes, indicative of gene copy number variation, were seen among the sequenced genomes, suggesting a degree of plasticity and variation in gene family content. A number of examples of polymorphism in the copy number of segmental duplications of regions spanning multiple genes were seen, such as that seen on scaffold DS571330 that appears to have been duplicated many times in the Rahman strain. Segmental duplications previously described in the HM-1:IMSS genome [10] appear to be present in the HM-1A and HM-1B clones but to show differential copy number among the other sequenced genomes (Additional file 9). Genomic plasticity can be an important generator of diversity and may be an important mechanism of molecular evolution in Entamoeba. Genomic plasticity is an important form of genome diversity among Leishmania species and strains [40]. Extensive size variation among homologous chromosomes is seen in Entamoeba [11] and in other parasitic protozoa such as trypanosomes [41]. If we consider the Rahman duplication in scaffold DS571330, 25 duplications of a 12.4 kilobase region would add around 0.3 megabases to the chromosome containing scaffold DS571330, a significant contribution to chromosomal size variation among strains. It remains to be seen whether such plasticity is a feature of natural parasite lines or a phenomenon arising during strain axenization, which is known to affect genome content [42].

In conclusion, our results suggest that among E. histolytica parasites, genomic plasticity and recombination may be relatively more important mechanisms of generating genome diversity than single nucleotide mutation.

\section{Materials and methods}

Origins and growth of E. histolytica strains and extraction of genomic DNA

Several E. histolytica isolates were sequenced. Isolates PVBM08F, PVBM08B, 2592100, MS84-1373 and MS275030 were axenized specifically for genome sequencing. The other isolates studied here are long-established laboratory strains.

PVBM08F and PVBM08B represent two culture isolations from the same individual, from feces and an intestinal biopsy, respectively. The male patient had recently spent 2 months in Liberia and presented with bloody diarrhoea and mucus, consistent with amoebic dysentery, and was strongly seropositive for E. histolytica. The cultures were kindly provided by Dra. Simonetta Gatti, Laboratory of Parasitology, Foundation IRCCS Policlinico San Matteo, Pavia, Italy.

MS84-1373 and MS27-5030 were established using routine monthly samples from asymptomatic children that were part of a long-term study of amoebiasis in Mirpur, Dhaka in Bangladesh [43]. Isolate 2592100 was from a case of amoebic dysentery seen at a local hospital in Dhaka. These cultures were kindly provided by $\mathrm{Dr}$ Rashidul Haque, International Centre for Diarrhoeal Disease Research, Bangladesh.

All five cultures were established in Robinson's medium [44]; on arrival in London they were adapted to 
growth in LYSGM with 5\% adult bovine serum [45]. Cultures were then axenized via monoxenic cultures in LYI-S-2 (liver extract, yeast extract, iron, serum growth medium) with $15 \%$ adult bovine serum and Crithidia fasciculata as an associate, as described [44].

To generate large amounts of genomic DNA for genome sequencing, parasites were cultured axenically in LYI-S-2. Cells were grown to a high density and harvested, usually after 72 hours of growth. Cell cultures were centrifuged and the culture medium removed, then cells were washed twice in phosphate-buffered saline before lysis in $300 \mu \mathrm{l}$ of QIAGEN cell lysis buffer (QIAGEN, Crawley, UK). Genomic DNA was purified using a modified version of the $\mathrm{CTAB}$ extraction protocol described elsewhere [46,47], using two rounds of phenol:chloroform:isoamyl alcohol (25:24:1) extraction. DNA was re-suspended in nuclease-free water, ready for preparation of sequencing libraries.

\section{SOLiD library preparation and sequencing}

Purified genomic DNA was used to prepare SOLiD ${ }^{\mathrm{TM}}$ fragment libraries, according to the manufacturer's protocols (Life Technologies, Foster City, CA, USA). Briefly, genomic DNA was fragmented by sonication on a Covaris S2 sonicator (Covaris Inc., Woburn, MA, USA) and end-repaired in preparation for P1 and P2 adaptor ligation. Adaptors were ligated and the samples size selected and amplified by standard PCR. DNA was bound to SOLiD ${ }^{\mathrm{TM}} \mathrm{P} 1$ beads and amplified by emulsion PCR, followed by enrichment for templated beads. The DNA was 3' modified before deposition on the sequencing slide, ensuring attachment of the beads to the slide. All libraries were sequencing on $\mathrm{SOLiD}^{\mathrm{TM}} 4$ sequencer (Life Technologies), to produce $50 \mathrm{bp}$ reads.

\section{SOLiD read mapping}

Sequence reads were mapped to the genome sequence of E. histolytica strain HM-1:IMSS using the BurrowsWheeler Aligner (BWA) mapping software [48]. The reference sequence matched that in data release 1.3 of the AmoebaDB database [49]; its 1,496 scaffolds contained 20,734,772 bases (excluding unknown bases represented by ' $\mathrm{N}$ '). Mapping parameters allowed up to four colorspace mismatches (that is, equivalent to up to two SNPs) per 48 bp read (after trimming off 5' and 3' terminal color calls), which represents up to approximately $4.2 \%$ divergence from the reference. The alignments were filtered to retain only uniquely aligned reads. These alignments were used for all further analyses.

\section{Analysis of repetitiveness of the reference genome}

Non-unique mapping to the reference genome will lead to gaps in coverage of the genome. In order to assess mapping to the reference, the relative repetitiveness and uniqueness of the reference genome was estimated. The reference sequence was used to generate $50 \mathrm{bp}$ sequence fragments representing the entire sequence, each offset from the last by $1 \mathrm{bp}$. These fragments were mapped to the reference genome using the Bowtie v0.12.1 short read mapper [50], allowing 0 mismatches. The number of times each fragment exactly matched a genomic region was recorded and the proportions of reads mapped to $1,2,3$ to 10,11 to 100 and > 100 different positions were calculated.

\section{Analysis of SNP variants}

Nucleotide variants between mapped strain and reference were called separately for each strain using the SAMtools pileup software [51]. Bases were called for each strain using the MAQ algorithm, assuming a ploidy of 4 [11]. Putative SNP positions were filtered to remove those with coverage $<5$, coverage in the top $5 \%$ (to remove potential artifactual heterozygous SNPs caused by 'stacking-up' of paralogous sequence), SNP quality < 20 or mapping quality $<20$ (SNP and mapping quality scores are phred-scaled: 20 represents $99 \%$ accuracy of the call) or within $5 \mathrm{bp}$ of a putative insertion or deletion. The effect of varying these threshold parameters on the predicted number of SNPs (in the Rahman strain) was assessed (Additional file 3). An independently generated dataset of Roche 454 reads representing the Rahman genome (manuscript in preparation) was used to validate SNP calls from the SOLiD data (Additional file 3). These reads were mapped to the same reference genome as the SOLiD data. The mean coverage across SNPs was $24 \times$. All SNP calls were tabulated and are displayed in Additional file 2. In order to define a subset of high confidence candidate SNP markers to estimate a phylogeny and to test for evidence of recombination among strains, 3,696 SNPs that varied between strains but were putatively homozygous within each strain, occurred in coding sequences and had a base called in every strain were designated 'high quality candidate marker' SNPs (Additional file 4).

\section{Estimating a phylogeny of strains}

The 3,696 'high quality candidate marker' SNPs were used to estimate a phylogeny of the sequenced E. histolytica strains. E. histolytica-only phylogenies (based on 3,696 positions) were estimated using two phylogenetic approaches: distance-based analysis (neighbor-joining) and maximum likelihood (ML). Both methods were implemented in MEGA 5 [52]. ML used the General Time Reversible model of evolution, selected as best using the model testing function in MEGA 5, while neighbor-joining used the maximum composite likelihood model. Statistical support for distance and ML 
trees was evaluated using bootstrapping with 1,000 replicates.

In order to root these phylogenies, a set of $E$. dispar variants at orthologous positions was defined. This was done by aligning regions flanking E. histolytica SNP positions (100 bp on either side) to the $E$. dispar genome assembly using NUCmer [53] and filtering the alignments to remove any that were not unambiguous best alignments from both species. This resulted in a set of 1,897 positions at which an E. dispar base could be confidently determined. This set of positions was used to build a bootstrapped neighbor-joining phylogeny using programs from the PHYLIP software package [54], which placed the $E$. dispar branch along the branch linking PVBM08B and PVBM08F to the other E. histolytica strains. The $E$. histolytica-only phylogenies were rooted along this branch.

\section{Testing for evidence of recombination among lineages}

The four-gametes (or four-haplotypes) test was applied to test for evidence of recombination during the evolutionary history of the E. histolytica strains. Under the infinite sites model of evolution, nucleotide sites mutate only once in the evolutionary history of a sample of related sequences, with no subsequent or back-mutation. Therefore, the maximum number of possible haplotypes formed by a pair of nucleotide sites in the absence of recombination is three. Four haplotypes can only be produced by a recombination event occurring between the two positions. Moreover, if recombination has happened, the occurrence of four haplotypes should increase in frequency with greater distance between variable nucleotide sites.

In order to detect this, 100,000 pairs of polymorphic 'high quality candidate marker' positions on the same scaffold (that is, putatively physically linked) were randomly sampled and the proportion of pairs showing four haplotypes was calculated for 100 distance intervals corresponding to centiles of the distance (each of 1,000 pairs of polymorphic sites). The proportion of pairs showing four haplotypes was plotted against the median distance for each interval. The correlation between this proportion and the distance between positions was tested by Spearman's rank correlation.

To strengthen the conclusion of meiotic recombination based on the results of the four-haplotypes test, we carried out simulations of samples undergoing recombination or gene conversion to compare the expected patterns of polymorphism. Simulations were carried out using Hudson's 'ms' software [55], which generates samples of sequence by coalescent simulation. In this case, two mutations were simulated (randomly assigned to branches of the coalescent tree) 100,000 times. Recombination was modeled by specifying a recombination parameter (4.Ne.r, where Ne is the effective population size and $r$ is the per generation probability of a crossover occurring in the sequence). Gene conversion was modeled by specifying a parameter similar to the recombination parameter (4.Ne.f, where $f$ is the per generation probability of a gene conversion event in the sequence) as well as the length of the 'converted' region. The proportion of four-haplotype SNP pairs per centile interval (that is, 1,000 simulations) was plotted.

\section{Analysis of gene copy number variation}

In order to detect variation in gene copy number among strains (including duplicated and missing genes), read counts were estimated for each gene in each strain. These counts were normalized for different library sizes between strains and for different gene lengths by expressing each as RPKM. In order to detect copy number variation, each strain was compared to HM-1:IMSS (in this case, the average RPKM for the HM-1A and HM$1 B$ strains). Genes where the average HM1 RPKM was very high (top $12.5 \%$ ) or low (bottom $12.5 \%$ ) were not considered in the analysis, to avoid calling genes as missing when the lack of unique mapping is actually due to repetitiveness, leaving a set of 6,228 genes. Genes with RPKM values two-fold higher or two-fold lower were considered to have a variable number of copies. In addition, putative missing genes had to have an RPKM value of $<1$. The analysis was run both for the original alignments and for alignments filtered so that only reads with unique start positions were considered. This second analysis was done to remove the possibility that high coverage was due to PCR duplicates arising during sequencing library preparation. However, with short read fragment libraries such as these, and particularly across short genes with deep coverage, many nonunique start positions are expected by chance, so removing them will dampen the signal of a high copy number. Therefore, the results of both analyses were shown.

Analysis of SNP polymorphism in gene coding sequences SNPs in the coding regions of genes were classified as nonsynonymous (amino acid-altering) or synonymous (causing no change to the protein sequence) using custom perl scripts. Counts of the numbers of homozygous nonsynonymous and synonymous SNPs in polymorphic genes were tabulated (Additional file 5).

\section{Data availability}

All sequence read data have been deposited in the EBI short read archive and are publicly available. All read data are labeled with the study accession number ERP001383 and individual samples are labeled with the following sample accession numbers: HM-1A, 
ERS132616; HM-1B, ERS132617; Rahman, ERS132618; 2592100, ERS132619; PVBM08B, ERS132620; PVBM08F, ERS132621; IULA:1092:1, ERS132622; HK-9, ERS132623; MS84-1373, ERS132624; MS27-5030, ERS132625.

\section{Additional material}

\section{Additional file 1: Coverage of genes in sequenced strains} Histograms of the proportion covered of E. histolytica genes. The proportions of E. histolytica genes with 0 to $10 \%, 11$ to $20 \%, 21$ to $30 \%$, 31 to $40 \%, 41$ to $50 \%, 51$ to $60 \%, 61$ to $70 \%, 71$ to $80 \%, 81$ to $90 \%$ and 91 to 100\% of their sequence covered are plotted. The majority of genes are well covered (91 to $100 \%$ of their length) by sequence libraries.

Additional file 2: Table of single nucleotide variants in $E$. histolytica strains relative to the HM-1:IMSS reference genome. SNP calls relative to the HM-1:IMSS reference genome.

Additional file 3: Additional analyses carried out to test the effects of SNP calling parameters, verify called SNPs and to simulate the four-haplotypes test. Additional analyses carried out to test the effects of varying the SNP calling parameters on the numbers of SNPS called in the Rahman strain, verify the called SNPs in Rahman strain by comparison with an independently generated dataset and to simulate the four-haplotypes test under models of varying recombination and gene conversion.

Additional file 4: Table of high quality candidate marker SNPs. Table of high quality candidate markers: defined as SNPs that vary between strains but are homozygous within each strain, occur in coding sequences and have a base called in every strain sequenced.

Additional file 5: Table of polymorphic genes in E. histolytica strains relative to the HM-1:IMSS reference genome. Counts of homozygous nonsynonymous and synonymous SNPs in polymorphic genes.

Additional file 6: Table of putative nonsense mutations in $E$. histolytica genes. Table of SNPs causing putative nonsense mutations in E. histolytica strains. The table includes both homozygous and heterozygous SNPS.

Additional file 7: Highly polymorphic genes in E. histolytica. Table of genes with five or more homozygous nonsynonymous SNPs in one or more strains, with details of protein features.

Additional file 8: Genes showing deep coverage in one or more $E$. histolytica strains. Table of genes (with RPKM values) with more than two-fold greater depth of coverage than the average of $\mathrm{HM}-1 \mathrm{~A}$ and $\mathrm{HM}$ $1 \mathrm{~B}$ in one or more strain, representing putative high copy number genes in that strain.

Additional file 9: Genes putatively missing from one or more $E$. histolytica strains. Table of genes (with RPKM values) with RPKM $<1$ and where this value is $<50 \%$ of the average of $\mathrm{HM}-1 \mathrm{~A}$ and $\mathrm{HM}-1 \mathrm{~B}$, representing putative missing genes in that strain.

\section{Abbreviations}

bp: base pair; Gal/GalNAc-lectin: galactose/N-acetyl-D-galactosamineinhibitable lectin; ML: maximum likelihood; MSP: merozoite surface protein; RPKM: reads per kilobase per million mapped reads; SNP: single nucleotide polymorphism; STIRP: serine: threonine and isoleucine rich protein.

\section{Acknowledgements}

This work was supported by a project grant from the Wellcome Trust. $\mathrm{NH}$ is supported by a Wolfson Merit Award from the Royal Society of Great Britain. We are grateful to Dra. Simonetta Gatti for providing parasite isolates PVBM08B and PVBM08F for axenization and to Dr Rashidul Haque and Dr Bill Petri for providing parasite isolates MS84-1373 and MS27-5030 (collected with funding from NIH grant no:2R01Al43596-13) for axenization. We also thank Miaomiao He for help with some analyses and two anonymous reviewers for their comments on the original manuscript.

\section{Author details}

${ }^{1}$ Institute of Integrative Biology, University of Liverpool, Biosciences building, Crown Street, Liverpool L69 7AH, UK. ²Pathogen Molecular Biology Department, Faculty of Infectious and Tropical Diseases, London School of Hygiene and Tropical Medicine, Keppel Street, London WC1E 7HT, UK. ${ }^{3}$ Bernhard Nocht Institute for Tropical Medicine, Bernhard-Nocht-Str. 74 20359 Hamburg, Germany.

\section{Authors' contributions}

Experiments were conceived and designed by NH, SP, and CGC. Analyses were carried out by GDW. Cell cultures of HM-1A and HM-1B were grown and DNA isolated by IB and ET; all other strains were grown and genomic DNA isolated by CGC and GDW. DNA sequencing libraries were made, and sequencing carried out, by SK and PK. The manuscript was drafted by GDW, with contributions from all authors. All authors read and approved the final manuscript for publication.

\section{Competing interests}

The authors declare that they have no competing interests.

Received: 14 November 2011 Revised: 29 April 2012

Accepted: 25 May 2012 Published: 25 May 2012

\section{References}

1. Pritt BS, Clark CG: Amebiasis. Mayo Clin Proc 2008, 83:1154-1159.

2. Nozaki T, Kobayashi S, Takeuchi T, Haghighi A: Diversity of clinical isolates of Entamoeba histolytica in Japan. Arch Med Res 2006, 37:277-279.

3. Haque R, Ali IM, Sack RB, Farr BM, Ramakrishnan G, Petri WA Jr: Amebiasis and mucosal lgA antibody against the Entamoeba histolytica adherence lectin in Bangladeshi children. J Infect Dis 2001, 183:1787-1793.

4. Walsh JA: Problems in recognition and diagnosis of amebiasis: estimation of the global magnitude of morbidity and mortality. Rev Infect Dis 1986, 8:228-238.

5. Diamond LS, Clark CG: A redescription of Entamoeba histolytica Schaudinn, 1903 (Emended Walker, 1911) separating it from Entamoeba dispar Brumpt, 1925. J Eukaryot Microbiol 1993, 40:340-344

6. Ali IK, Solaymani-Mohammadi S, Akhter J, Roy S, Gorrini C, Calderaro A, Parker SK, Haque R, Petri WA Jr, Clark CG: Tissue invasion by Entamoeba histolytica: evidence of genetic selection and/or DNA reorganization events in organ tropism. PLoS Negl Trop Dis 2008, 2:e219.

7. Blessmann J, Van Linh P, Nu PA, Thi HD, Muller-Myhsok B, Buss H, Tannich E: Epidemiology of amebiasis in a region of high incidence of amebic liver abscess in central Vietnam. Am J Trop Med Hyg 2002, 66:578-583.

8. Salit IE, Khairnar K, Gough K, Pillai DR: A possible cluster of sexually transmitted Entamoeba histolytica: genetic analysis of a highly virulent strain. Clin Infect Dis 2009, 49:346-353.

9. Loftus B, Anderson I, Davies R, Alsmark UC, Samuelson J, Amedeo P, Roncaglia P, Berriman M, Hirt RP, Mann BJ, Nozaki T, Suh B, Pop M, Duchene M, Ackers J, Tannich E, Leippe M, Hofer M, Bruchhaus I, Willhoeft U, Bhattacharya A, Chillingworth T, Churcher C, Hance Z, Harris B, Harris D, Jagels K, Moule S, Mungall K, Ormond D, et al: The genome of the protist parasite Entamoeba histolytica. Nature 2005, 433:865-868.

10. Lorenzi HA, Puiu D, Miller JR, Brinkac LM, Amedeo P, Hall N, Caler EV: New assembly, reannotation and analysis of the Entamoeba histolytica genome reveal new genomic features and protein content information. PLoS Negl Trop Dis 2010, 4:e716.

11. Willhoeft U, Tannich E: The electrophoretic karyotype of Entamoeba histolytica. Mol Biochem Parasitol 1999, 99:41-53.

12. Petri WA Jr, Haque R, Mann BJ: The bittersweet interface of parasite and host: lectin-carbohydrate interactions during human invasion by the parasite Entamoeba histolytica. Annu Rev Microbiol 2002, 56:39-64.

13. MacFarlane RC, Singh U: Identification of an Entamoeba histolytica serine-, threonine-, and isoleucine-rich protein with roles in adhesion and cytotoxicity. Eukaryot Cell 2007, 6:2139-2146.

14. Tanabe K, Mackay M, Goman M, Scaife JG: Allelic dimorphism in a surface antigen gene of the malaria parasite Plasmodium falciparum. J Mol Biol 1987, 195:273-287

15. Polley SD, Weedall GD, Thomas AW, Golightly LM, Conway DJ: Orthologous gene sequences of merozoite surface protein 1 (MSP1) from 
Plasmodium reichenowi and $P$. gallinaceum confirm an ancient divergence of $P$. falciparum alleles. Mol Biochem Parasitol 2005, 142:25-31

16. Jeffares DC, Pain A, Berry A, Cox AV, Stalker J, Ingle CE, Thomas A, Quail MA, Siebenthall K, Uhlemann AC, Kyes S, Krishna S, Newbold C, Dermitzakis ET, Berriman M: Genome variation and evolution of the malaria parasite Plasmodium falciparum. Nat Genet 2007, 39:120-125.

17. Ayeh-Kumi PF, Ali IM, Lockhart LA, Gilchrist CA, Petri WA Jr, Haque R: Entamoeba histolytica: genetic diversity of clinical isolates from Bangladesh as demonstrated by polymorphisms in the serine-rich gene. Exp Parasitol 2001, 99:80-88.

18. Escueta-de Cadiz A, Kobayashi S, Takeuchi T, Tachibana H, Nozaki T: Identification of an avirulent Entamoeba histolytica strain with unique tRNA-linked short tandem repeat markers. Parasitol Int 2010, 59:75-81.

19. Haghighi A, Kobayashi S, Takeuchi T, Masuda G, Nozaki T: Remarkable genetic polymorphism among Entamoeba histolytica isolates from a limited geographic area. J Clin Microbiol 2002, 40:4081-4090.

20. Samie A, Obi CL, Bessong PO, Houpt E, Stroup S, Njayou M, Sabeta C, Mduluza T, Guerrant RL: Entamoeba histolytica: genetic diversity of African strains based on the polymorphism of the serine-rich protein gene. Exp Parasitol 2008, 118:354-361

21. Zaki M, Reddy SG, Jackson TF, Ravdin Jl, Clark CG: Genotyping of Entamoeba species in South Africa: diversity, stability, and transmission patterns within families. J Infect Dis 2003, 187:1860-1869.

22. Beck DL, Tanyuksel M, Mackey AJ, Haque R, Trapaidze N, Pearson WR, Loftus B, Petri WA Jr: Entamoeba histolytica: sequence conservation of the Gal/GalNAc lectin from clinical isolates. Exp Parasitol 2002, 101:157-163.

23. Bhattacharya D, Haque R, Singh U: Coding and noncoding genomic regions of Entamoeba histolytica have significantly different rates of sequence polymorphisms: implications for epidemiological studies. J Clin Microbiol 2005, 43:4815-4819.

24. Ghosh S, Frisardi M, Ramirez-Avila L, Descoteaux S, Sturm-Ramirez K, Newton-Sanchez OA, Santos-Preciado Jl, Ganguly C, Lohia A, Reed S, Samuelson J: Molecular epidemiology of Entamoeba spp.: evidence of a bottleneck (Demographic sweep) and transcontinental spread of diploid parasites. J Clin Microbiol 2000, 38:3815-3821.

25. Smythe JA, Peterson MG, Coppel RL, Saul AJ, Kemp DJ, Anders RF: Structural diversity in the 45 -kilodalton merozoite surface antigen of Plasmodium falciparum. Mol Biochem Parasitol 1990, 39:227-234.

26. Thomas AW, Carr DA, Carter JM, Lyon JA: Sequence comparison of allelic forms of the Plasmodium falciparum merozoite surface antigen MSA2. Mol Biochem Parasitol 1990, 43:211-220.

27. Okenu DM, Thomas AW, Conway DJ: Allelic lineages of the merozoite surface protein 3 gene in Plasmodium reichenowi and Plasmodium falciparum. Mol Biochem Parasitol 2000, 109:185-188.

28. Trucco C, Fernandez-Reyes D, Howell S, Stafford WH, Scott-Finnigan TJ, Grainger M, Ogun SA, Taylor WR, Holder AA: The merozoite surface protein 6 gene codes for a $36 \mathrm{kDa}$ protein associated with the Plasmodium falciparum merozoite surface protein-1 complex. Mol Biochem Parasitol 2001, 112:91-101.

29. Ware LA, Kain KC, Sim Lee BK, Haynes JD, Baird JK, Lanar DE: Two alleles of the 175-kilodalton Plasmodium falciparum erythrocyte binding antigen. Mol Biochem Parasitol 1993, 60:105-109.

30. Cavanagh DR, Dodoo D, Hviid L, Kurtzhals JA, Theander TG, Akanmori BD, Polley S, Conway DJ, Koram K, McBride JS: Antibodies to the N-terminal block 2 of Plasmodium falciparum merozoite surface protein 1 are associated with protection against clinical malaria. Infect Immun 2004, 72:6492-6502.

31. Conway DJ, Cavanagh DR, Tanabe K, Roper C, Mikes ZS, Sakihama N, Bojang KA, Oduola AM, Kremsner PG, Arnot DE, Greenwood BM, McBride JS: A principal target of human immunity to malaria identified by molecular population genetic and immunological analyses. Nat Med 2000, 6:689-692.

32. Metzger WG, Okenu DM, Cavanagh DR, Robinson JV, Bojang KA, Weiss HA McBride JS, Greenwood BM, Conway DJ: Serum IgG3 to the Plasmodium falciparum merozoite surface protein 2 is strongly associated with a reduced prospective risk of malaria. Parasite Immunol 2003, 25:307-312.

33. Polley SD, Tetteh KK, Lloyd JM, Akpogheneta OJ, Greenwood BM, Bojang KA, Conway DJ: Plasmodium falciparum merozoite surface protein 3 is a target of allele-specific immunity and alleles are maintained by natural selection. J Infect Dis 2007, 195:279-287.
34. Osier FH, Murungi LM, Fegan G, Tuju J, Tetteh KK, Bull PC, Conway DJ, Marsh K: Allele-specific antibodies to Plasmodium falciparum merozoite surface protein-2 and protection against clinical malaria. Parasite Immunol 2010, 32:193-201.

35. Stanley SL Jr: Entamoeba histolytica genome: something old, something new, something borrowed and sex too? Trends Parasitol 2005, 21:451-453.

36. Ramesh MA, Malik SB, Logsdon JM Jr: A phylogenomic inventory of meiotic genes; evidence for sex in Giardia and an early eukaryotic origin of meiosis. Curr Biol 2005, 15:185-191.

37. Weedall GD, Sherrington J, Paterson S, Hall N: Evidence of gene conversion in genes encoding the Gal/GalNac lectin complex of Entamoeba. PLoS Negl Trop Dis 2011, 5:e1209.

38. Rougeron V, De Meeûs T, Kako Ouraga S, Hide M, Bañuls AL: "Everything you always wanted to know about sex (but were afraid to ask)" in Leishmania after two decades of laboratory and field analyses. PLOS Pathog 2010, 6:e1001004.

39. Birky CW Jr: Giardia sex? Yes, but how and how much? Trends Parasitol 2010, 26:70-74.

40. Rogers MB, Hilley JD, Dickens NJ, Wilkes J, Bates PA, Depledge DP, Harris D, Her Y, Herzyk P, Imamura H, Otto TD, Sanders M, Seeger K, Dujardin JC, Berriman M, Smith DF, Hertz-Fowler C, Mottram JC: Chromosome and gene copy number variation allow major structural change between species and strains of Leishmania. Genome Res 2011, 21:2129-2142.

41. Callejas S, Leech V, Reitter C, Melville S: Hemizygous subtelomeres of an African trypanosome chromosome may account for over $75 \%$ of chromosome length. Genome Res 2006, 16:1109-1118.

42. Mukherjee C, Clark CG, Lohia A: Entamoeba shows reversible variation in ploidy under different growth conditions and between life cycle phases. PLoS Negl Trop Dis 2008, 2:e281.

43. Haque R, Mondal D, Karim A, Molla IH, Rahim A, Faruque AS, Ahmad N, Kirkpatrick BD, Houpt E, Snider C, Petri WA Jr: Prospective case-control study of the association between common enteric protozoal parasites and diarrhea in Bangladesh. Clin Infect Dis 2009, 48:1191-1197.

44. Clark CG, Diamond LS: Methods for cultivation of luminal parasitic protists of clinical importance. Clin Microbiol Rev 2002, 15:329-341.

45. Stechmann A, Hamblin K, Pérez-Brocal V, Gaston D, Richmond GS, van der Giezen M, Clark CG, Roger AJ: Organelles in Blastocystis that blur the distinction between mitochondria and hydrogenosomes. Curr Biol 2008, 18:580-585.

46. Clark CG, Diamond LS: The Laredo strain and other 'Entamoeba histolytica-like' amoebae are Entamoeba moshkovskii. Mol Biochem Parasitol 1991, 46:11-18.

47. DNA Isolation. [http://entamoeba.lshtm.ac.uk/dnaisoln.htm].

48. Li H, Durbin R: Fast and accurate short read alignment with BurrowsWheeler transform. Bioinformatics 2009, 25:1754-1760.

49. Aurrecoechea C, Barreto A, Brestelli J, Brunk BP, Caler EV, Fischer S, Gajiria B, Gao X, Gingle A, Grant G, Harb OS, Heiges M, lodice J, Kissinger JC, Kraemer ET, Li W, Nayak V, Pennington C, Pinney DF, Pitts B, Roos DS, Srinivasamoorthy G, Stoeckert CJ Jr, Treatman C, Wang H: AmoebaDB and MicrosporidiaDB: functional genomic resources for Amoebozoa and Microsporidia species. Nucleic Acids Res 2011, 39:D612-D619.

50. Langmead B, Trapnell C, Pop M, Salzberg SL: Ultrafast and memoryefficient alignment of short DNA sequences to the human genome. Genome Biol 2009, 10:R25.

51. Li H, Handsaker B, Wysoker A, Fennell T, Ruan J, Homer N, Marth G, Abecasis G, Durbin R, 1000 Genome Project Data Processing Subgroup: The Sequence Alignment/Map format and SAMtools. Bioinformatics 2009, 25:2078-2079.

52. Tamura K, Peterson D, Peterson N, Stecher G, Nei M, Kumar S: MEGA5: molecular evolutionary genetics analysis using maximum likelihood, evolutionary distance, and maximum parsimony methods. Mol Biol Evol 2011, 28:2731-2739.

53. Delcher AL, Phillippy A, Carlton J, Salzberg SL: Fast algorithms for largescale genome alignment and comparision. Nucleic Acids Res 2002, 30:2478-2483.

54. PHYLIP. [http://evolution.genetics.washington.edu/phylip.html].

55. Hudson RR: Generating samples under a Wright-Fisher neutral model of genetic variation. Bioinformatics 2002, 18:337-338.

doi:10.1186/gb-2012-13-5-r38

Cite this article as: Weedall et al: Genomic diversity of the human intestinal parasite Entamoeba histolytica. Genome Biology 2012 13:R38. 